\title{
Nobiletin Inhibits Non-Small-Cell Lung Cancer by Inactivating WNT/ $\beta$-Catenin Signaling through Downregulating miR-15-5p
}

\author{
Sang Hyup Han, ${ }^{1,2}$ Jeong Hee Han, ${ }^{1,2}$ Wan Joo Chun, ${ }^{2}$ Sang Soo Lee, ${ }^{3}$ Hae Sung Kim $\mathbb{D}^{1,2}$ \\ and Jin Won Lee $\mathbb{D}^{1}$ \\ ${ }^{1}$ Department of Surgery, Chuncheon Sacred Heart Hospital, College of Medicine, Hallym University, \\ Chuncheon 24253, Gangwon-Do, Republic of Korea \\ ${ }^{2}$ Department of Pharmacology, College of Medicine, The Kangwon University of Korea, Chuncheon, Republic of Korea \\ ${ }^{3}$ Institute for Skeletal Aging \& Orthopedic Surgery, Hallym University-Chuncheon Sacred Heart Hospital, \\ Chuncheon, Republic of Korea \\ Correspondence should be addressed to Hae Sung Kim; biogra@hallym.ac.kr and Jin Won Lee; sdf119@hallym.or.kr
}

Received 4 November 2021; Revised 2 December 2021; Accepted 7 December 2021; Published 30 December 2021

Academic Editor: Qing Li

Copyright (c) 2021 Sang Hyup Han et al. This is an open access article distributed under the Creative Commons Attribution License, which permits unrestricted use, distribution, and reproduction in any medium, provided the original work is properly cited.

\begin{abstract}
Background. Nobiletin is a natural compound with anticancer activity; however, the mechanism is not clear. Methods. The inhibitory effect of nobiletin on non-small-cell lung cancer (NSCLC) cells was examined using soft agar, Transwell, and apoptosis analyses. Cancer stemness was measured by sphere assay. Genes and miRNAs regulated by nobiletin were identified by wholegenome sequencing. Protein levels were detected by western blot and immunofluorescence assays. Results. Nobiletin significantly inhibited NSCLC cell colony formation and sphere formation and induced apoptosis. Nobiletin upregulated negative regulators of $\mathrm{WNT} / \beta$-catenin signaling, including NKD1, AXIN2, and WIF1, while it inhibited the expression of $\beta$-catenin and its downstream genes, including c-Myc, c-Jun, and cyclin D1. Furthermore, we identified that GN inhibits miR-15-5p expression in NSCLC cells and that NKD1, AXIN2, and WIF1 are the target genes of miR-15-5p. Conclusions. Nobiletin has a strong inhibitory effect on NSCLC, and nobiletin plays an anticancer role by inhibiting miR-15-5p/ $\beta$-catenin signaling in NSCLC.
\end{abstract}

\section{Introduction}

Lung cancer is the most frequently diagnosed malignancy and the leading cause of cancer-related mortality worldwide [1-3]. Non-small-cell lung cancer (NSCLC) accounts for $80-85 \%$ of all lung cancers. Despite considerable advancements in diagnosis and treatment modalities, including surgery and chemoradiation therapy, the survival of patients with advanced stages of the disease remains unsatisfactory. Several oncogenes and tumor suppressor genes known to be associated with NSCLC have been investigated to analyze tumor behavior and determine their prognostic significance [4-6]. In this regard, there is a need to discover new agents for the treatment of this deadly disease. Many kinds of natural products have been proven to possess anticancer properties [7-9]. These types of components can affect the overall process of carcinogenesis by several mechanisms. Among them, nobiletin has been known to have an anticancer effect, as it can inhibit the proliferation, invasion, or metastasis of tumor cells. Nobiletin is a flavonoid compound isolated from the peels of citrus fruits, such as Citrus unshiu (Satsuma mandarin) and Citrus sinensis. Studies show that nobiletin exhibits an anticancer role in various cancers, including skin, prostate, colon, and breast cancer [10-18]. However, the anticancer mechanism of nobiletin is still unclear.

WNT/ $\beta$-catenin signaling is a well-studied oncogenic pathway that plays an important role in the development and progression of various cancers. For example, there have been some reports that $\mathrm{WNT} / \beta$-catenin might enhance the motility of malignant cells and tumor invasion, such as in breast cancer, melanoma, and gastric cancer [19-23]. As a 
result, many investigators have focused on the potential effect of some substances, such as flavonoids, on cancer prevention and therapy by inhibiting the $\mathrm{WNT} / \beta$-catenin signaling pathway [1-3]. In fact, most reports show that inhibition of $\mathrm{WNT} / \beta$-catenin signaling is a useful strategy for cancer treatment.

In this study, we demonstrated that nobiletin significantly inhibits NSCLC growth, metastasis, and stemness and stimulates NSCLC cell apoptosis. In addition, we demonstrated that nobiletin plays its role by inactivating Wnt/ $\beta$-catenin signaling by upregulating the expression levels of WIF1, AXIN2, and NKD1. We also identified that nobiletin inhibits the expression of WIF1, AXIN2, and NKD1 by inhibiting miR-15-5p in NSCLC cells.

\section{Materials and Methods}

2.1. Reagents. Bicinchoninic acid (BCA) assay reagent was purchased from Bio-Rad (California, USA), Lipofectamine was purchased from Invitrogen (California, USA), the ECL (enhanced chemiluminescence) kit was purchased from Amersham Pharmacia (Buckinghamshire, UK), and the Immobilon-p membrane was purchased from Millipore (Massachusetts, USA). RPMI (Roswell Park Memorial Institute) 1640 medium, fetal bovine serum (FBS), TryPLEExpress enzyme, and Hank's balanced salt solution were obtained from Gibco (New York, USA).

2.2. Cell Lines and Cultures. Cell lines H460 and A549 were obtained from ATCC (Manassas, VA, USA), and these cells were cultured in DMEM (HyClone, Shanghai, China) supplemented with 10\% FBS (ScienCell Research Laboratories, California, USA) in a $5 \% \mathrm{CO}_{2}$ humidified atmosphere at $37^{\circ} \mathrm{C}$. The cells were then treated with $2.5 \mu \mathrm{M}$ nobiletin for 24 hours and then subjected to a soft agar assay. After 10 days, the cell cultures were treated with $10 \mu \mathrm{L}$ of CCK-8 solution for 2 hours and incubated at $37^{\circ} \mathrm{C}$ for the determination of cell viability. A microplate reader was used to obtain the optical density (OD) at $40 \mathrm{~nm}$. This research was approved by the Institutional Review Board of Chuncheon Sacred Heart Hospital.

2.3. Cell Viability and Soft Agar Assay. For the cell viability assay, H460 and H549 cells were seeded in 96-well plates at a density of 5000/well. The next day, the cells were treated with nobiletin or PBS for $48 \mathrm{~h}$, and cell viability was measured using a Cell Counting Kit-8 (MedChem Express, JN, USA). For the soft agar assay, cells were treated with the indicated concentration of nobiletin for $48 \mathrm{~h}$, and then, cells were resuspended in $0.5 \mathrm{~mL}$ of $0.35 \%$ agar (Sigma) in the growth medium at a density of 5000 cells/well in 6-well plates. The agar-cell mixture was plated on the top of a solid layer of $0.8 \%$ agar in the growth medium. Colonies were counted 14 days later.

2.4. In Vitro Migration and Invasion Assay. Cell migration and invasion assays were performed using a Transwell chamber. Cells were treated with the indicated concentration of nobiletin for $48 \mathrm{~h}$. Then, the cells were resuspended, and $2 \times 10^{4}$ cells in the serum-free medium were plated into the top chamber with or without gel coating. The cell growth medium with $10 \%$ FBS was added under the chamber. After $24 \mathrm{~h}$, cells on the upper sides of the inserts were removed using cotton, and cells on the surface of the bottom sides of the inserts were fixed with $4 \%$ paraformaldehyde, followed by staining with $0.5 \%$ crystal violet. The cells in five random microscopic fields were photographed using an inverted phase-contrast microscope and counted.

2.5. Sphere Formation Assay. Cells were treated with the indicated concentration of nobiletin for $48 \mathrm{~h}$, and then, a sphere formation assay was carried out as described in previous studies [17-20,24]. In brief, cells were plated in Petri dishes (Corning, Tewksbury, MA, USA) at a concentration of approximately $10^{4}$ cells per $\mathrm{ml}$ of the sphere-forming medium consisting of a 1:1 mixture of high-glucose DMEM and Ham's Nutrient Mixture F-12 (Euroclone, Siziano, Italy) supplemented with $20 \mathrm{ng} / \mathrm{ml} \mathrm{EGF}$ and $20 \mathrm{ng} / \mathrm{ml}$ recombinant FGFb, 1X Insulin-Transferrin-Selenium (Life Technologies, Carlsbad, CA, USA), $4 \mathrm{mg} / \mathrm{ml}$ bovine serum albumin (BSA, Sigma-Aldrich, Saint Louis, Missouri, USA), 2 mM glutamine (Euroclone), $50 \mathrm{U} / \mathrm{ml}$ penicillin, and $0.05 \mathrm{mg} / \mathrm{ml}$ streptomycin (Euroclone). After 6-7 days of growth, spheres were counted.

2.6. Luciferase Reporter Assay. The luciferase reporter assay was carried out as described by Fend [19].

2.7. Western Blot and RT-PCR Analyses. Western blot and RT-PCR analyses were carried out as described in previous studies [17-24]. For western blot analysis, equal amounts of whole-cell extracts were separated using SDS-PAGE, and the separated proteins were transferred to PVDF membranes. Membranes were incubated with the indicated primary and secondary antibodies. Protein bands were detected by enhanced chemiluminescence (Amersham Biosciences). For RT-PCR and real-time qRT-PCR analyses, total RNA was isolated from freshly dissected intestines using TRIzol reagent (Life Technologies), and complementary DAN synthesis was performed using an Omniscript Kit according to the manufacturer's instructions (QIAGEN). PCR amplification of miR-15-5p and RNU6 was performed with a specific primer set obtained from Thermo Fisher Scientific. PCR amplification of the following genes was performed with the QuantiTect SYBR Green method. The sequences of the primer pairs were as follows: GAPDH forward, $5^{\prime}$ GGAGCGAGATCCCTCCAAAAT- $3^{\prime}$ and reverse, $5^{\prime}$ GGCTGTTGTCATACTTCTCATGG-3`; WIF1 forward, $5^{\prime}$-GTGTGAAATCAGCAAATGCC-3', and reverse, $5^{\prime}$ GTCTTCCATGCCAACCTTCT-3; AXIN2 forward, $5^{\prime}$ AATTCGCGGGAGGGGGC- $3^{\prime}$, and reverse, $5^{\prime}$ CTTCGTCGTCTGCTTGGTCAC-3 ' ${ }^{\prime}$ NKD1 forward, $5^{\prime}$ TCGCCGGGATAGAAAACTACA- $3^{\prime}$, and reverse, $5^{\prime}$ CAGTTCTGACTTCTGGGCCAC-3 '. 


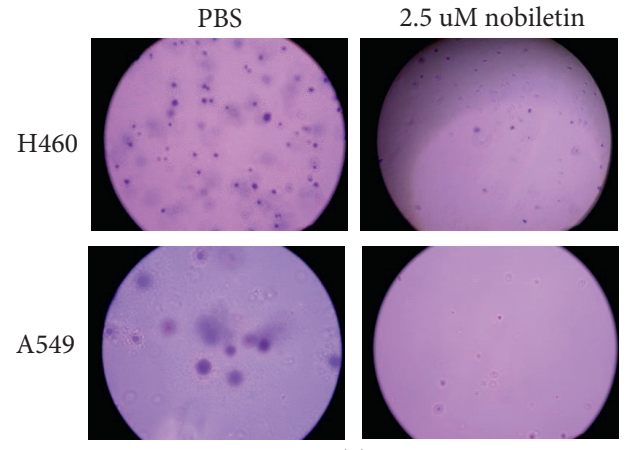

(a)
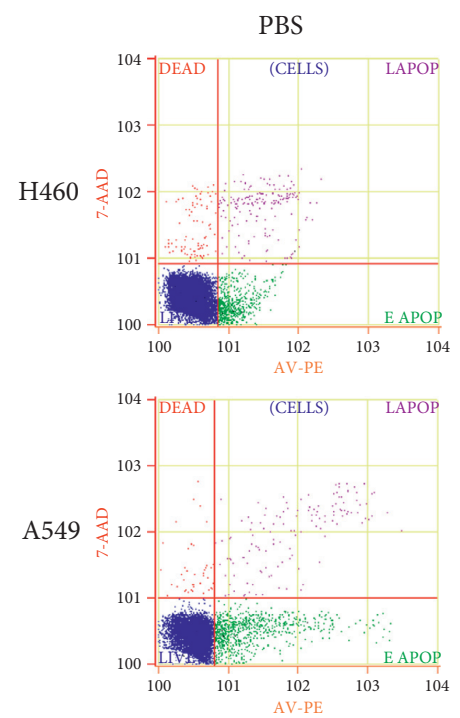
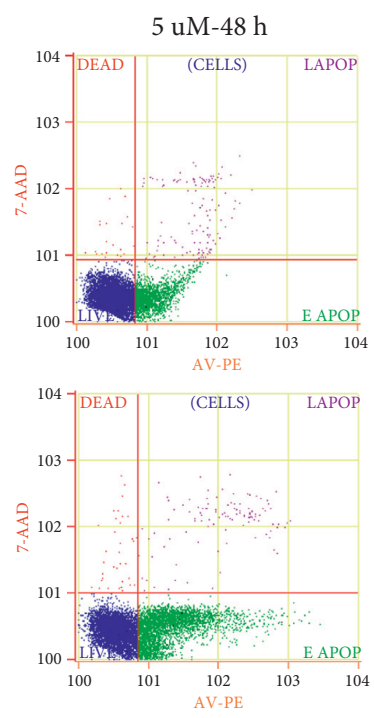

(c)

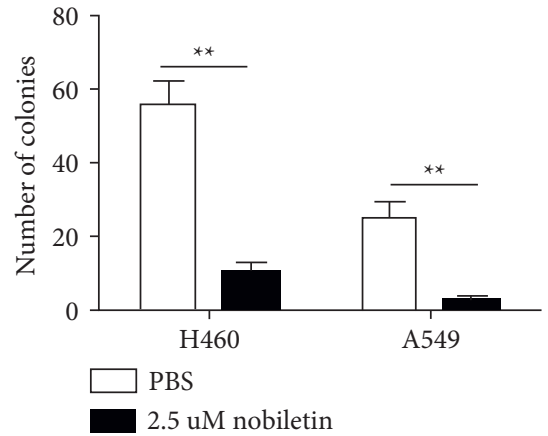

(b)

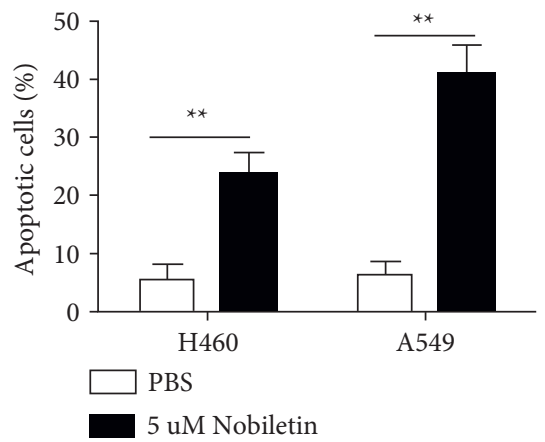

(d)

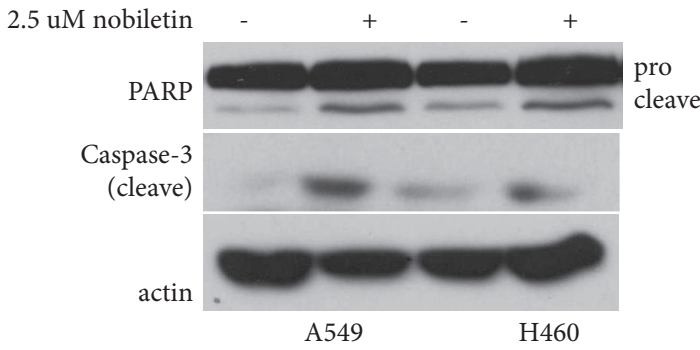

(e)

FIGURE 1: Nobiletin significantly inhibited NSCLC. $(\mathrm{a}, \mathrm{b})$ The inhibitory effects of nobiletin on NSCLC were measured by soft agar assay. $(b, c)$ Apoptosis-promoting effects of nobiletin were measured by flow cytometry assay. (d) The effects of nobiletin on the expression of proapoptotic proteins were measured by western blot. ${ }^{* *} P<0.01$.

2.8. Statistical Analysis. All data are presented as the mean \pm standard deviation (SD), and significant differences $(P<0.05)$ between groups were analyzed by Student's $t$-test using SAS statistical software version 6.12 (SAS Institute).

\section{Results}

3.1. Nobiletin Inhibited the Growth of NSCLC In Vitro. We first examined the inhibitory effects of nobiletin on NSCLC. Both H460 and A549 NSCLC cells were treated with nobiletin and then subjected to soft agar and apoptosis analyses. Our results showed that nobiletin significantly inhibited soft agar colony formation (Figures 1(a) and 1(b)) and significantly increased apoptosis in NSCLC cells (Figures 1(c) and 1(d)). Furthermore, western blot analysis confirmed the apoptosispromoting effects of nobiletin on NSCLC. Western blot analysis showed that nobiletin significantly increased proapoptotic protein levels, including cleaved PARP and cleaved caspase 3 (Figure 1(e)). Taken together, our findings suggested that nobiletin inhibits NSCLC by stimulating apoptosis.
3.2. Nobiletin Inhibited the Invasion and Migration of NSCLC. Metastasis is a poor prognostic factor of NSCLC. Therefore, we investigated the effect of nobiletin on the invasion and migration of A549 and H460 cells. Transwell chamber assays were conducted to evaluate the effects of nobiletin on invasion and migration. As shown in Figures 2(a) and 2(b), nobiletin significantly suppressed the invasive capacities of both A549 $(P<0.01)$ and H460 $(P<0.05)$ NSCLC cells compared to their control group. Consistently, migration assays also showed that nobiletin significantly inhibited the migration abilities of both A549 and H460 cells (Figures 2(c) and 2(d)), suggesting that nobiletin has powerful antimetastatic effects.

3.3. Nobiletin Inhibits WNT/ $\beta$-Catenin Signaling in NSCLC. To investigate the anticancer molecular mechanism of nobiletin in NSCLC, A549 and H460 cells were treated with nobiletin for 48 hours and then subjected to whole-genome sequencing. Nobiletin treatment altered the expression levels of many genes in both NSCLC cell lines (Figure 3(a)). In addition, Kyoto 



(a)

(b)
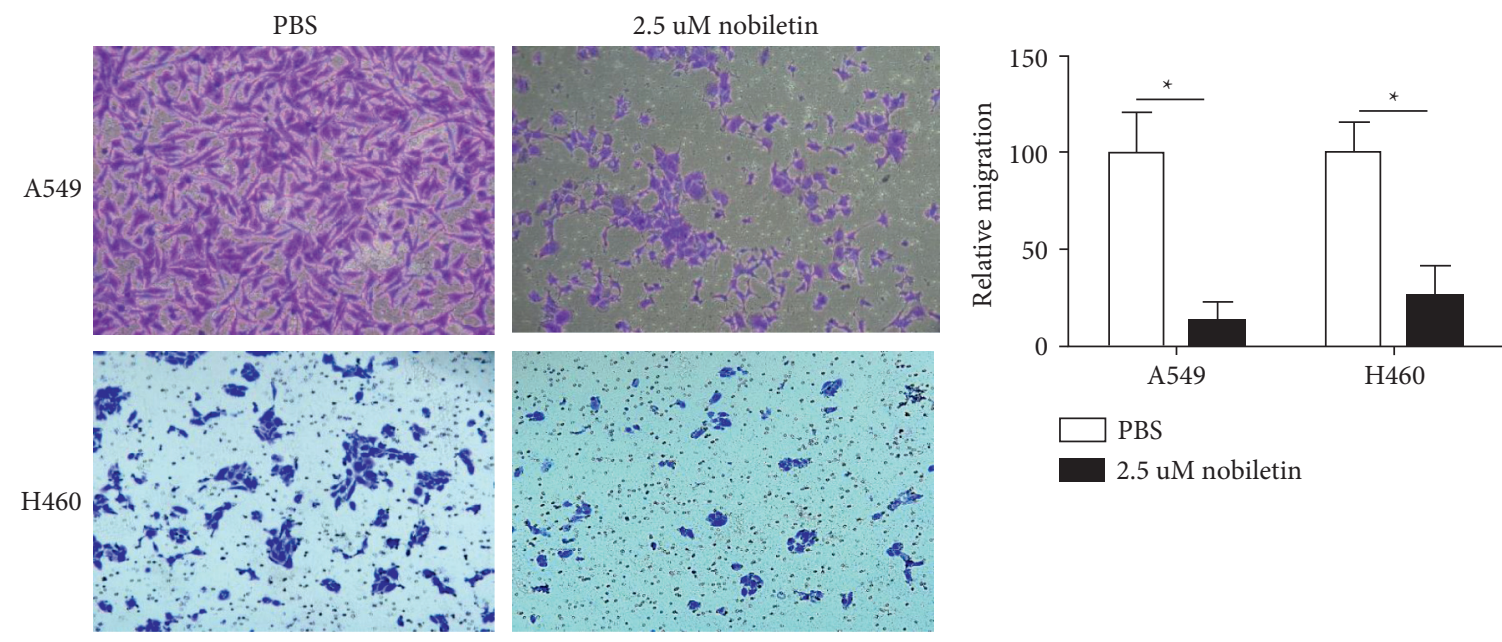

(c)

(d)

FIGURE 2: Nobiletin significantly inhibited the metastasis of NSCLC cells. (a, b) The effect of nobiletin on NSCLC cell invasion was measured. (c, d) The effect of nobiletin on NSCLC cell migration was measured. ${ }^{*} P<0.05$; ${ }^{*} P<0.01$.

Encyclopedia of Genes and Genomes (KEGG) analysis showed that nobiletin treatment significantly affected cancer stemness maintenance-related WNT/ $\beta$-catenin signaling (Figure 3(b)). In fact, bioinformatics analysis of mRNA sequencing results showed that nobiletin upregulated negative regulators of $\mathrm{WNT} / \beta$-catenin signaling, including NKD1, AXIN2, and WIF1 while inhibiting positive regulators of WNT/ $\beta$-catenin signaling, including WNT6 and Jun (Table 1). Consistently, gene set enrichment analysis (GSEA) showed that nobiletin treatment was negatively correlated with epithelial-mesenchymal transition (EMT) and cancer stemness in NSCLC (Figure 3(c)). Together, these findings suggested that nobiletin has anticancer effects by inhibiting WNT/ $\beta$-catenin signaling.

3.4. Nobiletin Inhibits Cancer Stemness and WNT/ $\beta$-Catenin Signaling in NSCLC. To investigate whether nobiletin is directly involved in the inhibition of cancer stemness and
$\mathrm{WNT} / \beta$-catenin signaling, NSCLC cells were treated with nobiletin and then subjected to sphere formation assays, and the expression levels of marker proteins of cancer stemness and WNT/ $\beta$-catenin signaling were detected. Our results showed that nobiletin significantly inhibited sphere formation of NSCLC cells (Figure 4(a)) and cancer stemness-related marker protein expression (Figure 4(b)). In addition, nobiletin treatment significantly inhibited $\beta$-catenin levels (Figures 4 (c) and $4(\mathrm{~d})$ ) and downstream protein expression levels of WNT/ $\beta$-catenin signaling (Figure 4(d)). These results suggested that nobiletin is directly involved in the inhibition of cancer stemness and the WNT/ $\beta$-catenin signaling pathway in NSCLC.

3.5. Nobiletin Suppressed WNT/ $\beta$-Catenin Signaling by Downregulating miR-15-5p Expression in NSCLC. To investigate the inhibitory mechanism of nobiletin on WNT/ $\beta$-catenin signaling, we performed miRNA sequencing using nobiletin-treated NSCLC cells and their control cells because previous studies showed that miRNAs were involved in the 


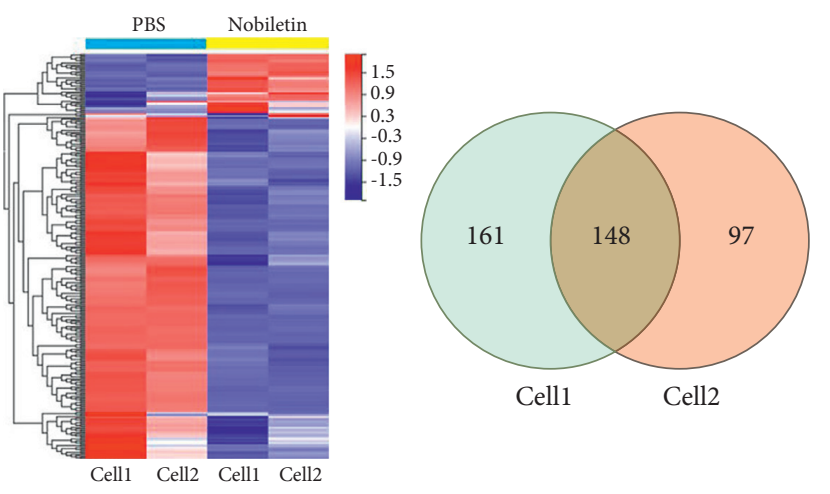

(a)

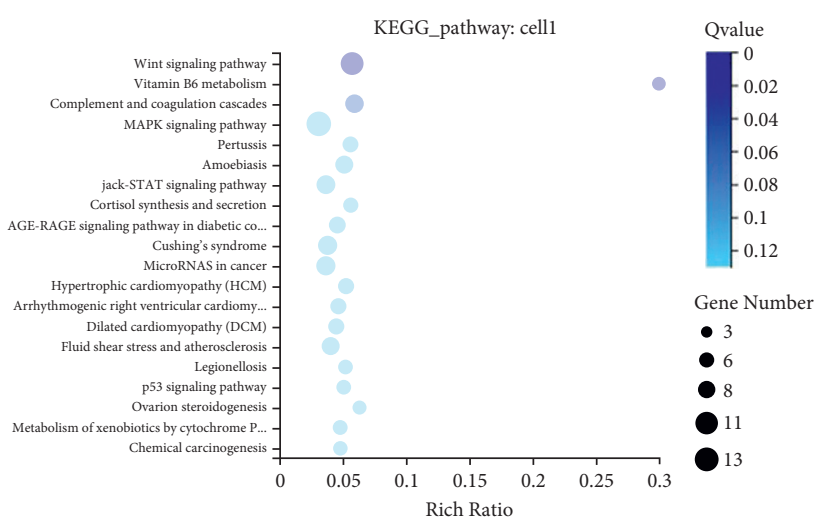

(b)

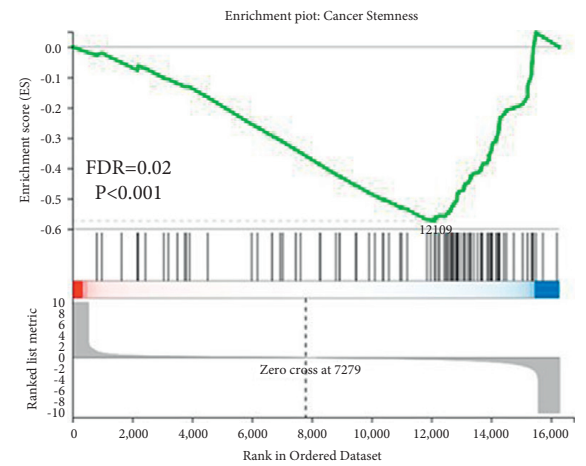

(c)

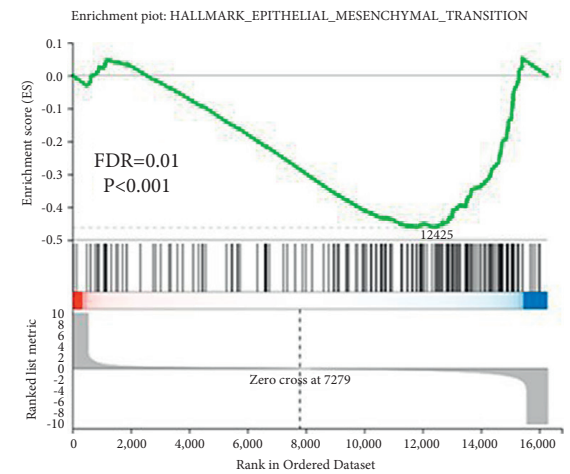

(d)

FIGURE 3: Nobiletin significantly affected WNT/ $\beta$-catenin signaling in NSCLC. (a) Heatmap showing that nobiletin treatment caused alterations in the expression levels of many genes in A594 and H460 cells. (b) Kyoto Encyclopedia of Gene and Genomes (KEGG) analysis revealed that nobiletin treatment significantly affected cancer stemness maintenance-related WNT/ $\beta$-catenin signaling. (c). GSEA showed that nobiletin treatment negatively correlated with epithelial-mesenchymal transition (EMT) and cancer stemness in NSCLC (d).

TABLE 1: Key genes related to WNT signaling that were affected by nobiletin treatment.

\begin{tabular}{lcc}
\hline Gene & Expression & Role in WNT signaling \\
\hline NKD1 & $U p$ & Inhibitor \\
AXIN2 & $U p$ & Inhibitor \\
WIF1 & $U p$ & Inhibitor \\
DKK1 & $U p$ & Inhibitor \\
WNT6 & Down & Activator \\
JUN & Down & Activator, downstream gene \\
\hline
\end{tabular}

regulation of $\mathrm{WNT} / \beta$-catenin signaling by targeting their regulators [25]. miRNA sequencing data showed that the expression levels of many miRNAs were affected by nobiletin treatment (Figures 5(a) and 5(b)). Among them, we chose miR-15-5p for further experiments (Figure 5(b)) because miR-15-5p was inhibited by nobiletin, and miRNA 

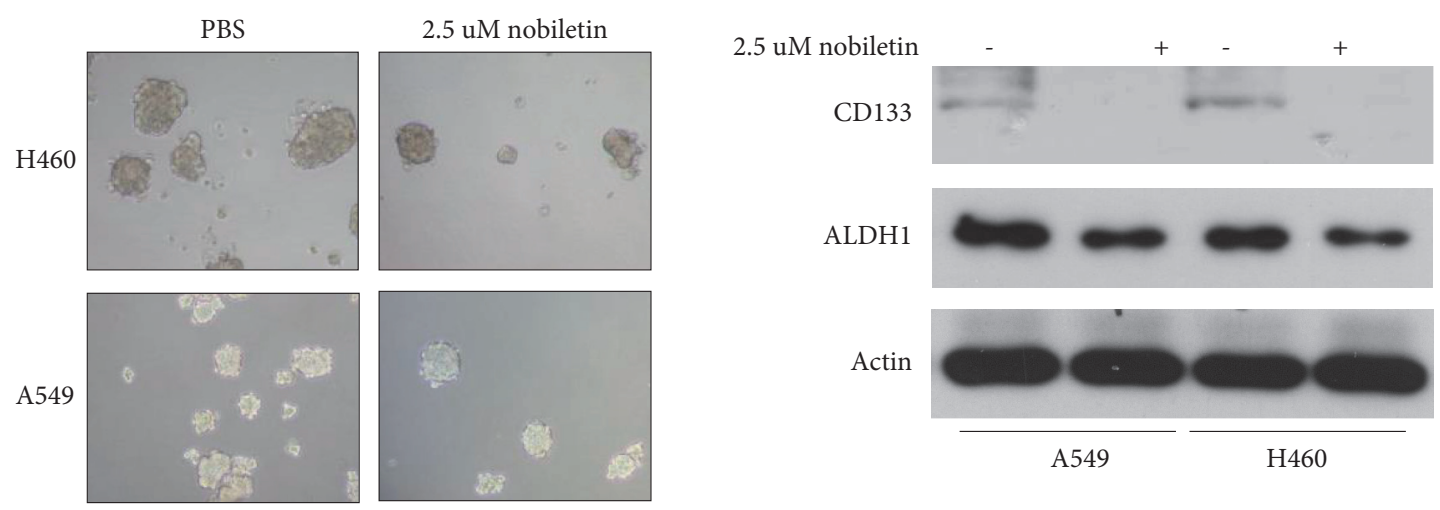

(a)
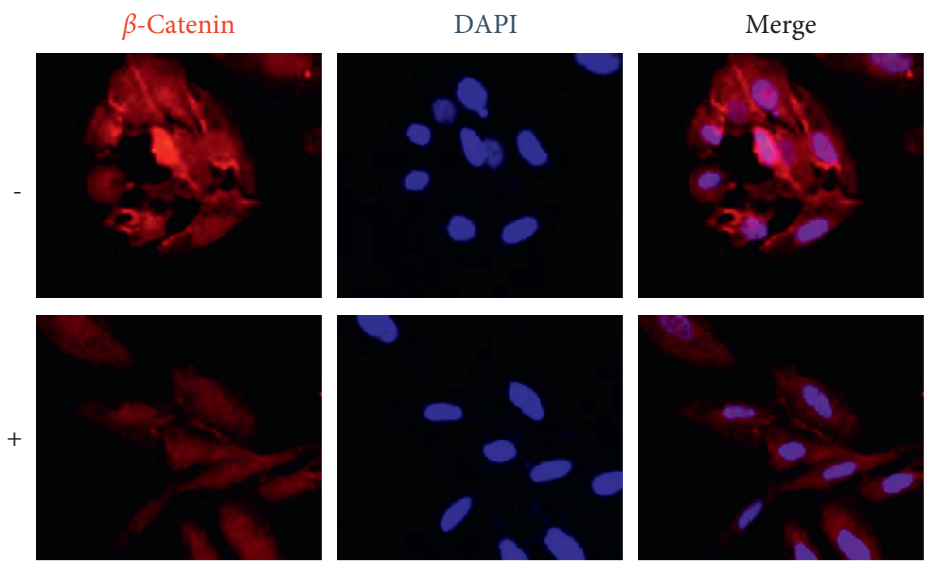

A549

$2.5 \mathrm{uM}$ nobiletin, $24 \mathrm{~h}$

(c)



(b)

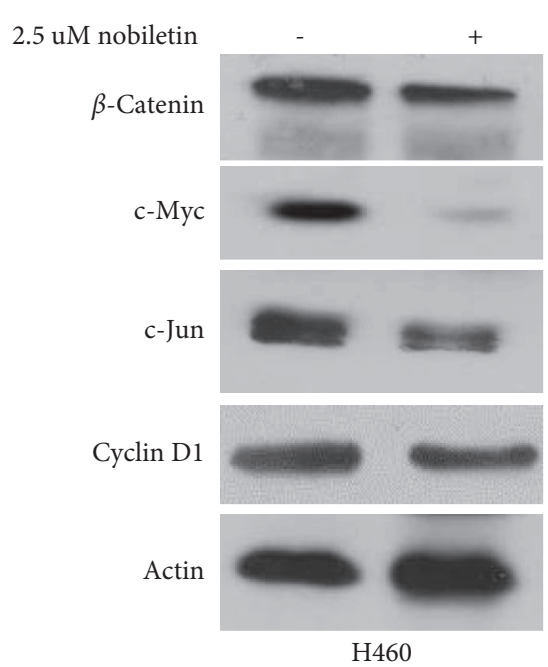

(d)

FIGURE 4: Nobiletin significantly inhibited cancer stemness and WNT/ $\beta$-catenin signaling in NSCLC cells. (a) The effect of nobiletin on NSCLC cell stemness was investigated using a sphere formation assay. (b) The effect of nobiletin on NSCLC cell stemness was investigated by measuring cancer stemness marker protein expression by western blot. (c) The effect of nobiletin on WNT/ $\beta$-catenin signaling was investigated by measuring $\beta$-catenin expression by IF. (d) The effect of nobiletin on WNT/ $\beta$-catenin signaling was investigated by measuring the downstream gene expression of $\beta$-catenin signaling by western blot.

database analysis showed that miR-15-5p can target negative regulators of $\mathrm{WNT} / \beta$-catenin signaling, including NKD1, AXIN2, and WIF1 (Figure 5(c)). First, we confirmed the inhibitory effect of nobiletin on miR-15-5p expression by qRT-PCR. As expected, nobiletin treatment significantly inhibited miR-15-5p expression in NSCLC cell lines (Figure 5(d)). In addition, a luciferase reporter assay showed that overexpression of miR-15-5p inhibited the luciferase expression regulated by the $3^{\prime}$ UTRs of NKD1, AXIN2, and WIF1 (Figure 5(e)). Notably, qRT-PCR and western blot analysis showed that overexpression of miR-15-5p (Figure 5(f)) significantly inhibited NKD1, AXIN2, and WIF1 expression at both the mRNA (Figure 5(g)) and protein levels (Figure 5(h)).

Next, we investigated whether miR-15-5p was involved in nobiletin-induced inhibition of WNT/ $\beta$-catenin signaling. As shown in Figures 6(a) and 6(b), inhibition of miR-15-5p inhibited the $\beta$-catenin expression level, while overexpression of miR-15-5p increased the $\beta$-catenin expression level. Notably, overexpression of miR-15-5p blocked nobiletin-induced inhibition of $\beta$-catenin expression (Figure 6(c)), suggesting that nobiletin inhibits $\mathrm{WNT} / \beta$-catenin signaling through inhibition of miR-15-5p in NSCLC.

\section{Discussion}

In recent decades, natural products have received widespread attention as anticancer drugs. They have anticancer effects and fewer side effects [1-4]. However, natural products have not been widely used in clinical treatment because their mechanism of action is not clear. Nobiletin is a ubiquitous flavonoid derived from the peel of citrus fruits. Previous studies have demonstrated that nobiletin possesses therapeutic and biological activities, including antioxidant, anticancer, cardioprotective, and anti-inflammatory effects $[10,11,13-18,24]$, but the anticancer mechanism is still unclear.

Cancer stemness is a key factor for cancer progression. Studies show that increased cancer stemness stimulates cancer cell resistance to chemotherapy and enhances cancer cell invasiveness, thereby contributing to cancer progression [26], and increased cancer stemness also stimulates EMT [25]. EMT is a process in which epithelial tumor cells lose their cell 


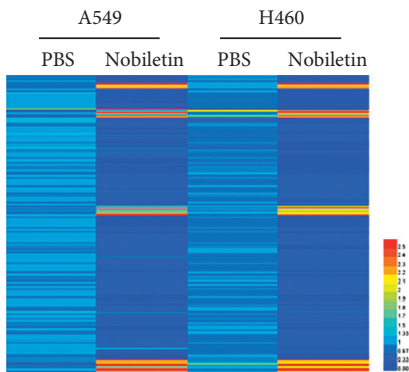

(a)

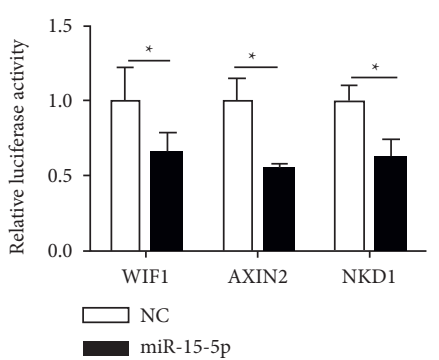

(e)

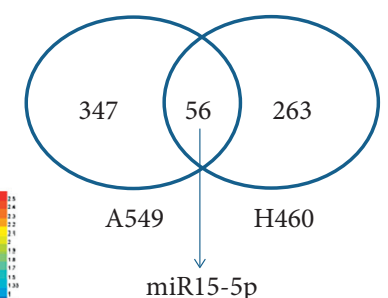

(b)

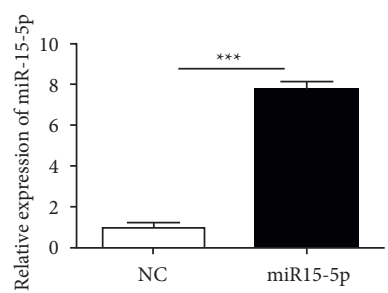

(f)

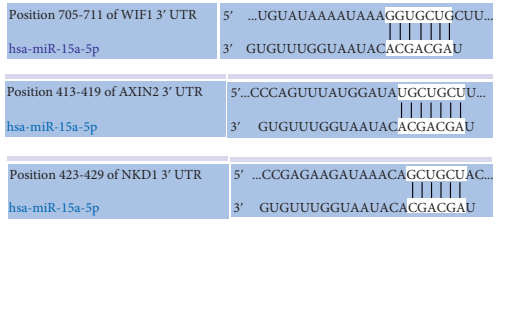

(c)

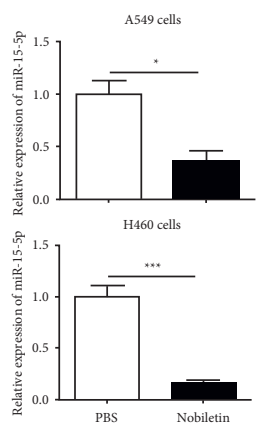

(d)



(g)

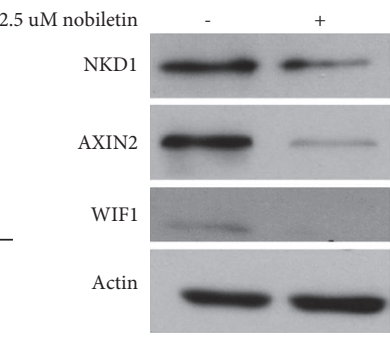

(h)

FIGURE 5: Nobiletin upregulates the negative regulator expression of WNT/ $\beta$-catenin signaling and miR-15-5p expression. (a) Heatmap showing that nobiletin affected the expression levels of many miRNAs in NSCLC cells. (b) Nobiletin inhibited miR-15-5p expression in both A549 and H460 cells. (c) Predicted binding sites of miR-15-5p in the $3^{\prime}$-UTRs of NKD1, AXIN2, and WIF1. (d) The inhibitory effect of nobiletin on miR-15-5p expression was measured by qRT-PCR in NSCLC cells. (e) Luciferase activity of the reporter driven by the $3^{\prime}$-UTRs of NKD1, AXIN2, and WIF1 in A549 cells cotransfected with negative control oligonucleotides (NC) or miR-15-5p. (f) The transfection efficacy of miR-15-5p was measured by qRT-PCR. (g) The effects of miR-15-5p on the mRNA expression levels of NKD1, AXIN2, and WIF1 were measured by qRT-PCR. (h) The effects of miR-15-5p on the protein expression levels of NKD1, AXIN2, and WIF1 were measured by western blot.

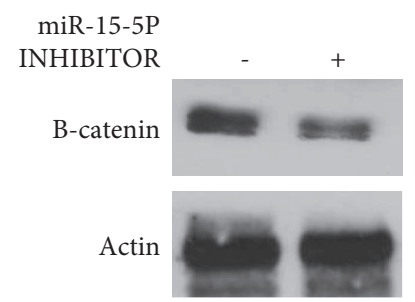

(a)

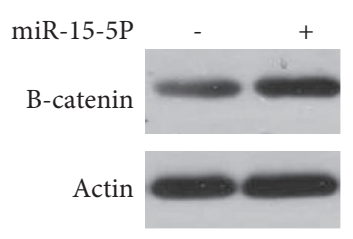

(b)



(c)

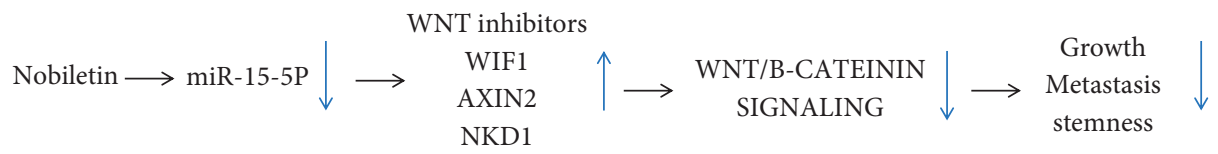

(d)

FIGURE 6: Nobiletin inhibits WNT/ $\beta$-catenin signaling by inhibiting miR-15-5p. (a, b) The negative regulation of $\beta$-catenin expression by miR-15-5p was measured by western blot. (c) Western blot showing that miR-15-5p overexpression blocked nobiletin-induced inhibition of $\beta$-catenin expression in A549 cells. (d) A schematic model of the regulation of NSCLC progression by nobiletin.

polarity and cell-cell adhesion so that tumor cells gain migratory and invasive properties [27]. During EMT, epithelial cells lose epithelial markers, such as E-cadherin, which is the main molecule of stable epithelial adherens junctions, so that the invasion-metastasis cascade is promoted, resulting in the exit of tumor cells from the primary site, invasion to adjacent organs, intravasation, and distant metastasis via the blood or lymphatic system. Studies have shown that inhibition of cancer stemness and EMT can dramatically inhibit cancer progression
$[28,29]$. Abnormally increased cancer stemness and EMT are caused by many factors in cancer, and hyperactivation of $\mathrm{WNT} / \beta$-catenin signaling is one of them $[30,31]$. Here, our data showed that nobiletin inhibits NSCLC progression, cancer stemness, EMT, and $\mathrm{WNT} / \beta$-catenin signaling, suggesting that nobiletin exerts its anticancer effects by inhibiting WNT/ $\beta$-catenin signaling-regulated cancer stemness and EMT.

Here, we also demonstrated that $\mathrm{WNT} / \beta$-catenin signaling was inactivated by nobiletin. $\mathrm{WNT} / \beta$-catenin 
signaling is often hyperactivated in cancers by the downregulation of negative regulators, including NSCLC [32]. In addition, studies have shown that downregulated expression of the $\mathrm{WNT} / \beta$-catenin signaling pathway is promoted by dysregulated miRNA expression in cancer [33]. Interestingly, studies have shown that natural products play an anticancer role by altering miRNA expression [34-36]. In this study, we reported for the first time that nobiletin suppresses miR-15-5p expression, thereby causing upregulation of miR-15-5p target genes, including negative regulators of WNT/ $\beta$-catenin signaling, such as NKD1, AXIN2, and WIF1, which inactivate WNT/ $\beta$-catenin signaling.

\section{Conclusions}

In conclusion, nobiletin inhibits NSCLC by inhibiting cancer stemness and EMT by inactivating the WNT/ $\beta$-catenin signaling pathway in NSCLC. Nobiletin inhibited miR-15-5p expression, thereby increasing negative regulators of the $\mathrm{WNT} / \beta$-catenin signaling pathway and ultimately inactivating the $\mathrm{WNT} / \beta$-catenin signaling pathway. Our results shed light on the mechanism of the anticancer effect of nobiletin and provide more inspiration for physicians in treating patients with NSCLC.

\section{Data Availability}

All data generated or analyzed during this study are included within this article.

\section{Ethical Approval}

This study was approved by the Institutional Review Board of Chuncheon Sacred Heart Hospital (2020-09-002), which waived the need for informed consent for the use of patient data due to the retrospective nature of the study. All methods were performed in accordance with the relevant guidelines and regulations.

\section{Disclosure}

This work was based on doctoral dissertation research completed at Kangwon National University of Korea.

\section{Conflicts of Interest}

The authors declare that there are no conflicts of interest regarding the publication of this article.

\section{Authors' Contributions}

Hae Sung Kim contributed equally to this work. JWL proposed the topic and conceived and designed the study. SHH performed the experimental examinations, including PCR. JHH and WJC analyzed the data and helped with the interpretation. HSK and SSL collaborated with the corresponding author in the writing of the manuscript. All authors read and approved the final manuscript.

\section{References}

[1] J.-Y. Hung, Y.-L. Hsu, Y.-C. Ko et al., "Didymin, a dietary flavonoid glycoside from citrus fruits, induces Fas-mediated apoptotic pathway in human non-small-cell lung cancer cells in vitro and in vivo," Lung Cancer, vol. 68, no. 3, pp. 366-374, 2010.

[2] K.-I. Park, H.-S. Park, M.-K. Kim et al., "Flavonoids identified from Korean Citrus aurantium L. inhibit Non-Small Cell Lung Cancer growth in vivo and in vitro," Journal of Functional Foods, vol. 7, pp. 287-297, 2014.

[3] Z. Zhao, B. Liu, J. Sun et al., "Scutellaria flavonoids effectively inhibit the malignant phenotypes of non-small cell lung cancer in an Id1-dependent manner," International Journal of Biological Sciences, vol. 15, no. 7, pp. 1500-1513, 2019.

[4] Y. Sekido, S. Bader, F. Latif et al., "Molecular analysis of the von Hippel-Lindau disease tumor suppressor gene in human lung cancer cell lines," Oncogene, vol. 9, no. 6, pp. 1599-1604, 1994.

[5] J. Carretero, P. P. Medina, R. Pio, L. M. Montuenga, and M. Sanchez-Cespedes, "Novel and natural knockout lung cancer cell lines for the LKB1/STK11 tumor suppressor gene," Oncogene, vol. 23, no. 22, pp. 4037-4040, 2004.

[6] J. Lin, T. Sun, L. Ji et al., "Oncogenic activation of c-Abl in non-small cell lung cancer cells lacking FUS1 expression: inhibition of c-Abl by the tumor suppressor gene product Fus1," Oncogene, vol. 26, no. 49, pp. 6989-6996, 2007.

[7] N. Amado, D. Predes, M. Moreno, I. Carvalho, F. Mendes, and J. Abreu, "Flavonoids and wnt/ $\beta$-catenin signaling: potential role in colorectal cancer therapies," International Journal of Molecular Sciences, vol. 15, no. 7, pp. 12094-12106, 2014.

[8] S. Mohana, M. Ganesan, N. Rajendra Prasad, D. Ananthakrishnan, and D. Velmurugan, "Retracted article: Flavonoids modulate multidrug resistance through wnt signaling in P-glycoprotein overexpressing cell lines," $B M C$ Cancer, vol. 18, no. 1, p. 1168, 2018.

[9] N. G. Amado, B. F. Fonseca, D. M. Cerqueira, V. M. Neto, and J. G. Abreu, "Flavonoids: potential Wnt/beta-catenin signaling modulators in cancer," Life Sciences, vol. 89, no. 15-16, pp. $545-554,2011$.

[10] A. Hermawan and H. Putri, "Bioinformatics studies provide insight into possible target and mechanisms of action of nobiletin against cancer stem cells," Asian Pacific Journal of Cancer Prevention, vol. 21, no. 3, pp. 611-620, 2020.

[11] S. S. Kesharwani, P. Mallya, V. A. Kumar, V. Jain, S. Sharma, and S. Dey, "Nobiletin as a molecule for formulation development: an overview of advanced formulation and nanotechnology-based strategies of nobiletin," AAPS PharmSciTech, vol. 21, no. 6, p. 226, 2020.

[12] J. Moon, L. Manh Hung, T. Unno, and S. Cho, "Nobiletin enhances chemosensitivity to adriamycin through modulation of the AKT/GSK3 $\beta / \beta$-catenin/MYCN/MRP1 signaling pathway in A549 human non-small-cell lung cancer cells," Nutrients, vol. 10, no. 12, p. 1829, 2018.

[13] H. Jiang, H. Chen, C. Jin, J. Mo, and H. Wang, "Nobiletin flavone inhibits the growth and metastasis of human pancreatic cancer cells via induction of autophagy, G0/G1 cell cycle arrest and inhibition of NF-kB signalling pathway," Journal of B. U. ON.: Official Journal of the Balkan Union of Oncology, vol. 25, no. 2, pp. 1070-1075, 2020.

[14] M. Ashrafizadeh, A. Zarrabi, S. Saberifar et al., "Nobiletin in cancer therapy: how this plant derived-natural compound targets various oncogene and onco-suppressor pathways," Biomedicines, vol. 8, no. 5, p. 110, 2020. 
[15] C. X. Lin, C. W. Tu, Y. K. Ma et al., "Nobiletin inhibits cell growth through restraining aerobic glycolysis via PKA-CREB pathway in oral squamous cell carcinoma," Food Sciences and Nutrition, vol. 8, no. 7, pp. 3515-3524, 2020.

[16] C. Da, Y. Liu, Y. Zhan, K. Liu, and R. Wang, "Nobiletin inhibits epithelial-mesenchymal transition of human nonsmall cell lung cancer cells by antagonizing the TGF- $\beta 1 /$ Smad3 signaling pathway," Oncology Reports, vol. 35, no. 5, pp. 2767-2774, 2016.

[17] P.-P. He, Q.-Q. Shen, M. Wen et al., "Nobiletin reduces LPLmediated lipid accumulation and pro-inflammatory cytokine secretion through upregulation of miR-590 expression," Biochemical and Biophysical Research Communications, vol. 508, no. 1, pp. 97-101, 2019.

[18] H. Huang, L. Li, W. Shi et al., "The multifunctional effects of nobiletin and its metabolites in vivo and in vitro," Evidencebased Complementary and Alternative Medicine: eCAM, vol. 2016, Article ID 2918796, 14 pages, 2016.

[19] L. Feng, J.-f. Zhang, L. Shi et al., "MicroRNA-378 suppressed osteogenesis of MSCs and impaired bone formation via inactivating wnt/ $\beta$-catenin signaling," Molecular TherapyNucleic Acids, vol. 21, pp. 1017-1028, 2020.

[20] R. Wu, B. Zhao, X. Ren et al., "MiR-27a-3p targeting GSK3 $\beta$ promotes triple-negative breast cancer proliferation and migration through wnt/ $\beta$-catenin pathway," Cancer Management and Research, vol. 12, pp. 6241-6249, 2020.

[21] Z. Wei, Y. Wang, L. Jiang et al., "miR-223 regulates oral squamous cell carcinoma metastasis through the Wnt/ $\beta$-catenin signaling pathway," Oral Oncology, vol. 109, Article ID 104941, 2020.

[22] M. Pehlivan, M. Soyoz, B. Cerci, H. I. K. Coven, Z. Yuce, and H. O. Sercan, "sFRP1 expression induces miRNAs that modulate Wnt signaling in chronic myeloid leukemia cells," Molecular Biology, vol. 54, no. 4, pp. 563-569, 2020.

[23] L. Jiang, X. Liu, A. Kolokythas et al., "Downregulation of the Rho GTPase signaling pathway is involved in the microRNA138-mediated inhibition of cell migration and invasion in tongue squamous cell carcinoma," International Journal of Cancer, vol. 127, no. 3, pp. 505-512, 2010.

[24] N. Yoshimizu, Y. Otani, Y. Saikawa et al., "Anti-tumour effects of nobiletin, a citrus flavonoid, on gastric cancer include: antiproliferative effects, induction of apoptosis and cell cycle deregulation," Alimentary Pharmacology \& Therapeutics, vol. 20, pp. 95-101, 2004.

[25] Y. Lei, L. Chen, and G. Zhang, "MicroRNAs target the Wnt/ $\beta$ catenin signaling pathway to regulate epithelialmesenchymal transition in cancer (Review)," Oncology Reports, vol. 44, no. 4, pp. 1299-1313, 2020.

[26] L. T. H. Phi, I. N. Sari, Y. G. Yang et al., "Cancer stem cells (CSCs) in drug resistance and their therapeutic implications in cancer treatment," Stem Cells International, vol. 2018, Article ID 5416923, 16 pages, 2018.

[27] N. A. Gloushankova, I. Y. Zhitnyak, and S. N. Rubtsova, "Role of epithelial-mesenchymal transition in tumor progression," Biochemistry Biokhimiia, vol. 83, no. 12-13, pp. 1469-1476, 2018.

[28] V. Ramesh, T. Brabletz, and P. Ceppi, "Targeting EMT in cancer with repurposed metabolic inhibitors," Trends in Cancer, vol. 6, no. 11, pp. 942-950, 2020.

[29] F. M. Davis, T. A. Stewart, E. W. Thompson, and G. R. Monteith, "Targeting EMT in cancer: opportunities for pharmacological intervention," Trends in Pharmacological Sciences, vol. 35, no. 9, pp. 479-488, 2014.
[30] Y. Zhang and X. Wang, "Targeting the Wnt/ $\beta$-catenin signaling pathway in cancer," Journal of Hematology \& Oncology, vol. 13, no. 1, p. 165, 2020.

[31] M. Koni, V. Pinnarò, and M. F. Brizzi, "The Wnt signalling pathway: a tailored target in cancer," International Journal of Molecular Sciences, vol. 21, no. 20, p. 7697, 2020.

[32] F. de Sousa Melo and L. Vermeulen, "Wnt signaling in cancer stem cell biology," Cancers, vol. 8, no. 7, p. 60, 2016.

[33] S. Okay, S. Yiping, and W. Thomas, "Downregulated MicroRNA-200a in meningiomas promotes tumor growth by reducing E-cadherin and activating the $\mathrm{Wnt} / \beta$-catenin signaling pathway," Molecular Therapy, vol. 17, p. S391, 2009.

[34] Q. Lin, L. Ma, Z. Liu et al., "Targeting microRNAs: a new action mechanism of natural compounds," Oncotarget, vol. 8, no. 9, pp. 15961-15970, 2017.

[35] B. Zhang, L. Tian, J. Xie, G. Chen, and F. Wang, "Targeting miRNAs by natural products: a new way for cancer therapy," Biomedicine \& Pharmacotherapy, vol. 130, Article ID 110546, 2020.

[36] F. Ahmed, B. Ijaz, Z. Ahmad, N. Farooq, M. B. Sarwar, and T. Husnain, "Modification of miRNA Expression through plant extracts and compounds against breast cancer: mechanism and translational significance," Phytomedicine, vol. 68, Article ID 153168, 2020. 